Poster Abstracts - EuroMedLab Munich 2021 - Munich, April 10-14, 2022 • DOI 10.1515/cclm-2021-5029

Neonatal and paediatric laboratory medicine, including prenatal testing

W073

\title{
OPTIMIZATION IN THE SCREENING OF CONGENITAL HYPOTHYROIDISM
}

\author{
J.A. Delgado $^{1}$, J.M. BauÇa ${ }^{1}$, G. PÉrez Esteban ${ }^{1}$, J. Robles Bauza ${ }^{1}$ \\ ${ }^{1}$ Department of Laboratory Medicine, Hospital Universitari Son Espases, Palma de Mallorca, Spain
}

\section{BACKGROUND-AIM}

Congenital primary hypothyroidism $(\mathrm{CH})$ is a thyroid hormone-deficiency syndrome in the newborn. Detection strategies and algorithms are diverse, as well as different thyrotropin cut-off values exist both for confirmation tests and to request a new sample. The aim of this study was to assess our protocol in terms of specificity and improve our screening process by optimizing thyrotropin cut-off values.

\section{METHODS}

Retrospective observational study between 2013-2019. All newborn children with a confirmation test for $\mathrm{CH}$ were considered for the study.

A ROC curve analysis was performed for thyrotropin cut-off value optimization in DBS which triggers a confirmatory test, and odds ratios were calculated.

An increase in the TSH cut-off value for confirmation would imply the request of a second DBS sample rather than a serum sample for confirmation. Therefore, diagnosis would be slightly delayed.

For individuals affected by the cut-off value modification, serum free thyroxine in the confirmation test were analyzed in terms of clinical outcomes.

\section{RESULTS}

A total of 72,133 newborn children were screened for $\mathrm{CH}$, and 208 individuals were included in the study. Incidence in our population was 1:2,000 live births.

The area under the ROC curve was 0.819 (CI95\%: 0.748-0.897). While the current cut-off value (thyrotropin $\geq 10 \mathrm{mIU} /$ L) had a specificity of $31.8 \%$ [ORs: 3.5 (CI95\%: 1.4-8.8)], the optimal cut-off value (thyrotropin $\geq 15 \mathrm{mIU} / \mathrm{L})$ yielded a specificity of $92.4 \%$ for the detection of $\mathrm{CH}$ and transient hypothyroidism [ORs: 15.9 (CI95\%: 7.1-35.8)], with no loss of sensitivity.

The change in the TSH cut-off value for the serum confirmation test would have led to a delay in its performance for $111 / 208(53 \%)$ newborn children included in the study. Of these, $95(86 \%)$ were classified as transient hyperthyrotropinemia, $7(6 \%)$ as $\mathrm{CH}$, and $9(8 \%)$ as transient hypothyroidism. When examining fT4 values of individuals with a negative impact due to the change in the cut-off value, no case of severe hypothyroxinemia was detected.

\section{CONCLUSIONS}

While keeping a maximum sensitivity, optimization of cut-off values may be of great usefulness not only with regard to demand management, but also to reduce family stress, which is of especial relevance for newborn. 
Poster Abstracts - EuroMedLab Munich 2021 - Munich, April 10-14, 2022 • DOI 10.1515/cclm-2021-5029

Neonatal and paediatric laboratory medicine, including prenatal testing

W074

ASSESSMENT OF THE IMPLEMENTATION OF NON-INVASIVE PRENATAL DIAGNOSIS IN OUR HEALTH AREA

M.M. Calero Ruiz $^{1}$, A. Sáez-Benito Godino ${ }^{1}$, O. Diz Mellado ${ }^{1}$

${ }^{1}$ H.U.Puerta del Mar, Cádiz, Spain

\section{BACKGROUND-AIM}

The implementation of non-invasive prenatal diagnosis has the objective of continuous improvement in pregnancy care, by offering all pregnant populations a combined first-trimester screening for the detection of the most frequent chromosomal aneuploidies.

The introduction in recent years of a non-invasive prenatal test (DPNI), which detects free circulating DNA in maternal blood, represents an improvement in these screening programs, with a sensitivity greater than $99 \%$ and a false positive rate of less than $0,1 \%$.

The objetive is analyze the results of the non-invasive prenatal test from September 2019 to December 2020.

\section{METHODS}

Observational, retrospective study of NIPD carried out in our laboratory since its implantation.

Following PACAC recommendations, they are subsidiaries of the free circulating fetal DNA test:

a) risk cases $1 / 50$ to $1 / 270$ in the combined screening of the first trimester

b) cases of a history of a child with chromosomal disease.

The fetal DNA detection test in maternal blood is performed with the Harmony test, issuing a risk result whenever the DNA fraction is above $5 \%$.

\section{RESULTS}

During the study period, a total of 47 DPNI were performed, $87.2 \%$ (41 cases) from the combined first trimester screening and $12.8 \%$ ( 6 cases) due to a history of a child with chromosomopathy.

The results of this test are described:

Non invasive 46cases: Normal 44cases and abnormal 2cases (1 Down's syndrome and 1 Edward's syndrome)

There was only one case (2.08\%) that was not informative on 2 occasions due to a fraction of fetal DNA less than $5 \%$, but which resulted in a normal fetus after performing an invasive test.

The mean fetal DNA was $9.8 \%$, with no false positive or false negative cases after confirmation with invasive testing.

\section{CONCLUSIONS}

- The DPNI test meets the standards of the PACAC program since the detection rate is above $99 \%$, with a false positive rate of less than $1 \%$ and a non-informative rate $<5 \%$.

-The implantation of the DPNI has meant the reduction of invasive tests derived from the first trimester prenatal screening. Only in $4.3 \%$ ( 2 cases) have either a chorionic villus study or amniotic fluid been performed to confirm cases of pathological results of PND.

-The health care for our pregnant women has been improved, reducing the risk of abortion and other complications and improving the acceptance of the blood extraction test compared to more aggressive techniques. 
Poster Abstracts - EuroMedLab Munich 2021 - Munich, April 10-14, 2022 • DOI 10.1515/cclm-2021-5029

Neonatal and paediatric laboratory medicine, including prenatal testing

W075

ASSESSMENT OF THE PREGNANCY ANEUPLOIDY SCREENING PROGRAM DURING 2020

M.M. Calero Ruiz ${ }^{1}$, A. Sáez-Benito Godino ${ }^{1}$, J.M. Gutiérrez Romero ${ }^{1}$, O. Diz Mellado ${ }^{1}$

${ }^{1}$ H.U.Puerta del Mar, Cádiz, Spain

\section{BACKGROUND-AIM}

Prenatal detection of genetic abnormalities is one of the great challenges of current Fetal Medicine. The surpose is analyze the results of the chromosomal disease screening of our reference population during 2020 and detect if it meets the quality standards defined by the SEGO.

\section{METHODS}

Observational, retrospective study of the combined first-trimester and second-trimester pregnancy screenings requested from our laboratory during 2019.

The ultrasound marker, nuchal translucency (TN) were collected from the 12-week ultrasound recorded in the digital history of the patient. Biochemical markers, Pappa and free B-hcg were collected from SIL (Omega 3000). Said tests were performed in week 8-9 of gestation and by means of electrochemiluminescence sandwich immunoassay on the Cobas e411 analyzer (Roche). SdWlab software was used to calculate risk.

As quality control, the false positive rate, false negative rate, detection rate and the median of the multiples of the median (MoM) of the external quality control of the three markers were calculated.

\section{RESULTS}

During the study period, 1,354 screenings were requested, $96.4 \%$ (1305 cases) from the first trimester and only $3.6 \%$ (49 cases) from the second trimester.

For the first trimester screening program, a total of 1232 low-risk results (94.4\%), 42 high-risk results (3.2\%) and 31 cases of early extractions (2.4\%) were obtained, while for the second trimester program 47 cases (95.9\%) presented low risk and 2 cases of them (4.1\%) high risk.

The results of the confirmatory tests are described:

-First trimester 1305cases: Non-invasive test (DPNI) 34 cases (Normal 32 cases and Abnormal 2 cases), Invasive test 8 cases (all normal)

-Second trimester 49cases: Invasive test 2cases (all normal)

The false positive rate for the first quarter was $3.1 \%$ and for the second quarter it was $4.1 \%$. No false negative cases were detected and the detection rate was $99.9 \%$.

In the external quality control of the program, a median of MoM TN was observed that varied between -1 and 0.9; Pappa between -3.7 and 4.5 and free B-hcg between -11.3 and 1.6 .

\section{CONCLUSIONS}

- The screening program meets SEGO standards since the detection rate is above $95 \%$ and with a false positive rate of less than $5 \%$.

-The assessment by external quality control reports a calculation of the appropriate medians since they do not deviate more than $5-10 \%$ from the unit except for the determination of free B-hcg, requiring a reassessment of said determination.

-Combined screening is a valid method for the prenatal diagnosis of chromosomopathies, but it requires quality control and a continuous review of the program to detect any anomalies and impose corrective measures efficiently and quickly. 
Poster Abstracts - EuroMedLab Munich 2021 - Munich, April 10-14, 2022 • DOI 10.1515/cclm-2021-5029

Neonatal and paediatric laboratory medicine, including prenatal testing

W076

TEN YEARS OF PRENATAL SCREENING FOR DOWN'S SYNDROME IN A TERTIARY HOSPITAL.

M.A. Ballesteros Vizoso ${ }^{1}$, B. Badal Cogul ${ }^{1}$, P. Argente Del Castillo Rodriguez ${ }^{1}$, B. Garcia Garcia ${ }^{1}$, J. Bauça ${ }^{1}$, M.I. Pastor Garcia $^{1}$, I. Martin Navas ${ }^{1}$

${ }^{1}$ Department of Laboratory Medicine. Hospital Universitari Son Espases, Palma de Mallorca, Spain.

\section{BACKGROUND-AIM}

Down's syndrome (DS), or trisomy 21 (T21), is the most frequent aneuploidy as 1 in every 800 live births are affected with it and is the most prevalent cause of mental retardation. Trisomy 18 (T18) and trisomy 13 (T13) have a lower incidence, 1:7,500 and 1:15,000, respectively.

Different prenatal screening strategies have been used over the years to detect aneuploidies.

The purpose was to evaluate the evolution of prenatal screening in our hospital and present a proposal for its improvement.

\section{METHODS}

Retrospective descriptive study conducted among 37,971 pregnant women. Screening strategies were evaluated over two periods. First, between $2 / 2009-9 / 2013$, combined screening $(13,455)$ was used during the first trimester (nuchal translucency (NT), pregnancy-associated-plasma-protein-A (PAPP-A), and free $\beta$ eta-human chorionic gonadotropin( $\beta$ hCG)). During the second trimester, triple screening was performed (alpha-fetoprotein (AFP), $\beta$-hCG, and unconjugated estriol (UE3)). The positive risk cut-off for Down Syndrome (DS) was 1:250. Between 10/2013-12/2018, contingent screening $(16,971)$ was performed during the first trimester (NT and PAPP-A) with a positive risk for DS $\geq 1: 30$. In the second trimester, quadruple screening ( $A F P, \beta-h C G, u E 3$, and Inhibin $A$ ) was used for pregnant women with intermediate risk $(1: 31-1: 1,000)$ with a positive risk for $D S$ considered $\geq 1: 250$. The analytical platforms used were DELFIA $®$ Xpress $\AA$, Access 2 and Lifecycle ${ }^{\circledR}$.

\section{RESULTS}

93\% (CI95\%:88.3-97.7) sensitivity was obtained for a false positive rate (FPR) of 3.3\% using combined screening; and 88\% (CI95\%:78.0-97.0) with 2.2\% FPR with contingent screening; reducing invasive testing during the first trimester from $3.7 \%$ to $1.1 \%$.

\section{CONCLUSIONS}

Contingent screening has led to a decrease in invasive tests and a better performance in the first trimester. The addition of Inhibin A reduced the FPR by $1 \%$. Results could be improved by implementing a strategy which includes contingent screening with cell-free fetal DNA (cffDNA). 
Poster Abstracts - EuroMedLab Munich 2021 - Munich, April 10-14, 2022 • DOI 10.1515/cclm-2021-5029

Neonatal and paediatric laboratory medicine, including prenatal testing

W077

HEALTHCARE AND ECONOMIC IMPACT OF NON-INVASIVE PRENATAL TESTING (NIPT) IN ANEUPLOIDIES SCREENING

M. Diaz-Gimenez ${ }^{1}$, A. Pozo-Giraldez ${ }^{1}$, A. Hervas-Romero ${ }^{1}$, A. Lope-Martinez ${ }^{1}$, E. Rodriguez-Borja ${ }^{1}$

${ }^{1}$ Laboratory of Clinical Biochemistry and Molecular Pathology. Hospital Clínico Universitario de Valencia (Spain)

\section{BACKGROUND-AIM}

Genetic alterations are among the main causes $(12-15 \%)$ of fetal/neonatal morbidity and mortality which both have an elevated social and economic impact. Until 2018, screening for chromosomopathies (mainly trisomies 18 and 21) was performed through biochemical/combined risk calculation during the first (DP1) or second (DP2) trimester of pregnancy. Those cases with a risk $\geq 1: 270$ were confirmed by an invasive test (amniocentesis or chorionic villous sampling). Although sensitivity for DP1 is $85-95 \%$, in DP2 decreases to $65 \%$. This low performance for biochemical/ combined screening along with clinical complications related to invasive testing favoured the introduction of high sensitivity (>99\%) non-invasive prenatal testing (NIPT) based on fetal DNA in maternal blood. NIPT is strongly recommended in high risk patients (1:51 to 1:270) avoiding invasive tests as first election tests.

Our aim was to evaluate the efficiency of NIPT in economic terms and reduction of clinical complications related to invasive tests.

\section{METHODS}

Retrospective, observational and descriptive study of years 2014, 2016 and 2018. NIPT was implemented in 2018 in our laboratory for patients with risk among 1:51 to 1:270 whereas invasive tests remained the confirmatory test when risk was higher than 1:50. Data analysed were: Total DP1 and DP2 performed in our Laboratory; Patients with a risk higher than 1:50; Patients with a risk between 1:51 and 1:270; NIPT performed (in 2018). For every high risk case, invasive tests performed, positivity for aneuploidies and clinical complications were also collected. Besides, for efficiency purposes, economic costs and ratio cost/patient were calculated and compared taking into account test cost in Euros (DP1 \& DP2: 166,68€; TPNI: $240 €$; Invasive test: $527,96 €)$.

\section{RESULTS}

Total DP1+DP2 in 2014 was 1642. From these, 13 had a risk $\geq 1: 50$ and 38 between 1:51 and 1:270. In 20161627 DP1+DP2 were performed, 18 with a risk $\geq 1: 50$ and 58 between 1:51 and 1:270. Finally, in 2018, total DP1+DP2 was 1442 being 17 of risk $\geq 1: 50$ and 58 among 1:51 and 1:270. Total TPNI in 2018 was 137 (higher than expected given that some requests were allowed outside the agreed protocol). Invasive tests performed in 2014, 2016 and 2018 were 41,43 and 20 respectively and clinical complications registered were 6 in 2014, 1 in 2016 and 0 in 2018. Regarding economic costs, cost/patient ratio was $182 €, 190 €$ and $183 €$ in 2014, 2016 and 2018 respectively.

\section{CONCLUSIONS}

Implementation of NIPT in the algorithm of aneuploidies screening has produced an important reduction of invasive procedures as well as a decrease of related clinical complications without any substantial increase in cost/patient ratio. 
Neonatal and paediatric laboratory medicine, including prenatal testing

W078

CORRELATION BETWEEN MATERNAL SERUM BIOCHEMICAL MARKERS WITH NONINVASIVE PRENATAL TESTING (NIPT) FOR PRENATAL SCREENING OF FETAL CHROMOSOMAL ABNORMALITIES .

V. Isufaj $^{1}$, I. Babamusta ${ }^{1}$

${ }^{1}$ American Hospital , Laboratory Department, Tirane - Albania

\section{BACKGROUND-AIM}

Prenatal screening for chromosomal abnormalities can be done by biochemical screening test like dual marker test ( DMT ) , triple marker test ( TMT ) and quadruple marker test (QMT ) .It is important to identify ideal screening test among them wich best correlates with result of Noninvasive Prenatal Testing (NIPT) which is confirmatory test of foetal chromosomal abnormalities. These helps to decrease need for invasive prenatal tests for foetal karyotyping. These study aims to evaluate sensitivity, specificity , diagnostic accuracy and correlation of dual marker test (DMT) with the result of Noninvasive Prenatal Testing (NIPT).

\section{METHODS}

The data were studied for 6 months in the Central Laboratory of the American Hospital from 1-st January 2019 to 30 June 2019 in women with unique pregnancy, non In vitro fertilization ( IVF ).

\section{RESULTS}

From the 1453 woman screened by biochemical marker tests, 1386 ( $95.3 \%$ ) were screen negative and 67 ( $4.7 \%$ ) women were screen positive. From these 67 women that resulted- with High Risk we have data only on 38 patients who performed Noninvasive Prenatal Testing (NIPT). In 35 women, it was false positive (92.1\%) and in 3 women true positive $(7.9 \%)$. In 1385 women the test results were true negative $(99.92 \%)$, but in one case, result was fals negative $(0.08 \%)$. Dual marker test (DMT) had higher sensitivity and specificity, more than $90 \%$.

\section{CONCLUSIONS}

Universal screening of antenatal women, irrespective of their age and parity is suggested. Dual marker test has highest diagnostic value and best correlation with the result of Noninvasive Prenatal Testing (NIPT) . 
Neonatal and paediatric laboratory medicine, including prenatal testing

W079

PRIMARY ADRENAL INSUFFICIENCY IN A BOY WITH INCREASED VERY LONG CHAIN FATTY ACIDS

\author{
$\underline{\text { I.M. Portell Rigo }}^{1}{ }^{\text {, J. Robles BauzÁ }}{ }^{2}$, J.M. BauÇÀ RossellÓ ${ }^{2}$, G. PÉrez Esteban ${ }^{2}$
}

${ }^{1}$ Clinical Analysis Unit; Biotechnology Department, Poniente Hospital Public Health Agency. El Ejido. Almeria, Spain.

${ }^{2}$ Clinical Analysis Unit; Biotechnology Department, Son Espases University Hospital. Palma de Mallorca. Islas Baleares, Spain.

\title{
BACKGROUND-AIM
}

$X$-linked adrenoleukodystrophy (X-ALD) is a genetic storage disorder caused by mutations in the ABCD1 gene encoding the ALDp protein, which is involved in the transport of very long-chain fatty acids (VLCFA C $\geq 22$ ) from the cytosol to the peroxisome to be degraded by $\beta$-oxidation. ALDp deficiency causes an accumulation of VLCFA in plasma and tissues, mainly in the white matter of the brain, spinal cord, adrenal glands and testes.

The clinical spectrum is variable, with most affected males presenting with adrenal insufficiency and myeloneuropathy. About $30 \%$ of them develop progressive cerebral ALD. Diagnostic suspicion is based on the presence of suggestive clinical findings (neurological and adrenal insufficiency) and neuroimaging. Confirmation requires biochemical analysis (elevated VLCFA, by means of LC-MS/MS) and genetic mutation study.

Describe the unexpected finding and diagnosis of X-ALD.

\section{METHODS}

Case description.

\section{RESULTS}

A 6-year-old male, with no family history of interest, was seen in Neuropediatrics for learning difficulties and poor school performance. He came to the emergency department for episodes of spatial disorientation with a blank stare and no verbal response for a few seconds.

Physical examination revealed visuospatial disorientation and slow response. An electroencephalogram revealed a slow wave pattern and a brain magnetic resonance imaging (MRI) showed areas of active demyelination of the white matter with a pattern compatible with X-ALD.

Laboratory tests and results:

-Corticotropin (ACTH) >1250 pg/ml (Reference value (RV): 1-46), cortisol $7.30 \mu \mathrm{gg} / \mathrm{dl}$ (RV: 1.73-14.82).

-Plasma VLCFA ( $\mu \mathrm{mol} / \mathrm{L})$ : behenic acid $(C 22: 0) 57.9(\mathrm{RV}<92.6)$, lignoceric(C24:0) 92.6 (RV <78.8), hexacosanoic(C26:0) $3.830(\mathrm{RV}<0.980), \mathrm{C} 24 / \mathrm{C} 22$ ratio $1.599(\mathrm{RV}<0.990), \mathrm{C} 26 / \mathrm{C} 22$ ratio $0.066(\mathrm{RV}<0.021)$.

- Genetic study showed 1073 C>G mutation (p. Ser358*) in ABCD1.

\section{CONCLUSIONS}

In the case of mild neurological symptoms, the result of the brain MRI provided a diagnosis and was urgently confirmed as X-ALD, along, with the subsequent biochemical results obtained (elevated VLCFA profile and primary adrenal insufficiency) and the genetic study.

Good collaboration among healthcare professionals is essential to establish the correct diagnosis without delays, to initiate the most appropiate treatment for each case and to improve the prognosis as far as possible. 
Neonatal and paediatric laboratory medicine, including prenatal testing

W080

EMERGENCY MANAGEMENT OF RATIO SFLT-1/PLGF IN PREECLAMPSIA WITH AND WITHOUT FETAL GROWTH RESTRICTION

\author{
$\underline{\text { D. Fatela-Cantillo }}^{1}$, J. Montenegro-Martinez ${ }^{1}$, D. NuÑez-Jurado ${ }^{1}$, A. Garcia-Serrano ${ }^{1}$, J.M. Guerrero-Montavez ${ }^{1}$ \\ ${ }^{1}$ Virgen del Rocío Hospital
}

\title{
BACKGROUND-AIM
}

Preeclampsia (PE) and fetal growth restriction (FGR) are major causes of perinatal death and handicap in survivors. The changes are more severe in FGR associated with PE (PE/FGR) compared to those with FGR alone. Low placental growth factor (PIGF) and high soluble fms-like tyrosine kinase-1 (sFlt-1) levels in maternal circulation indicate placental dysfunction and are associated with subsequent development of PE, regardless of whether fetal growth is affected or not. Therefore they provide a valuable tool to clinicians to accelerate time to clinical diagnosis and minimize adverse maternal outcomes in women with PE.

The aim of this study was to evaluate the strength of the association of sFlt-1/PIGF ratio, PIGF and sFIt-1 with PE in patients with and without FGR, in an obstetric high-risk population with clinical signs of suspected PE in the second and third trimester prior to delivery.

\section{METHODS}

An observational, analytical, retrospective case-control study was carried out at our hospital, which included 134 pregnant women who attended the obstetric emergency department or outpatient gynecology consultations for suspected PE. Data was collected between August 2020 to February 2021. We selected a group of patients from this cohort who were classified into two groups: patients with PE and FGR (Group 1) and patients with PE without FGR (Group 2). Statistical analysis of the data was performed with the SPSS software.

\section{RESULTS}

Median values (IQR) for Group 1 ( $\mathrm{n}=17$ ) were: PLGF=75,56 $(48,95) \mathrm{pg} / \mathrm{mL}, \mathrm{sFlt}-1=8567(7728,5) \mathrm{pg} / \mathrm{mL}, \mathrm{sFlt}-1 / \mathrm{PLGF}$ ratio $=101,75(150,73)$ and median values (IQR) for Group $2(n=29)$ were: PLGF=150,9 $(92,67) \mathrm{pg} / \mathrm{mL}, \mathrm{sFlt}-1=10240$ $(8616,0) \mathrm{pg} / \mathrm{mL}$, sFlt-1/PLGF ratio= $62,37(115,9)$. In the management of our cohort, significant differences are observed in PLGF levels ( $p=0.005$ ) between both Group 1 and Group 2.

\section{CONCLUSIONS}

Close monitoring of pregnant women with FGR and PE diagnosed in the second and third trimester of pregnancy should assess such the sFIt-1/PLGF ratio as both sFIt-1 and PLGF levels separately, since it provides us with differentiated and useful information in the management of pregnant women with suspected PE. 
Neonatal and paediatric laboratory medicine, including prenatal testing

W081

PITFALLS IN THE IVA DIAGNOSTIC IN NEWBORNSCREENING AND IT'S SOLUTION

K. Schöngruber $^{1}$, W. Röschinger ${ }^{1}$, R. Arnecke ${ }^{1}$, E. Schuhmann ${ }^{1}$

${ }^{1}$ Labor Becker \& Kollegen

\section{BACKGROUND-AIM}

The paediatric guidelines list 19 target diseases that must be included in the newborn screening (NGS), a so-called sensitive addiction test. To reduce false positive results, second-tier tests, specific confirmatory tests, are added to the NGS. The NGS detects among others acylcarnitines(AC) and amino acids, which are separated by fragmentation in the tandem mass spectrometer. ACs with the same number of $\mathrm{C}$ atoms are detected below the same mass.

\section{METHODS}

In the case of increases above a defined value, a confirmation or differentiation is carried out via downstream secondtier tests, which run via an upstream HPLC column and the first separation already takes place before fragmentation and detection in the tandem MS.

\section{RESULTS}

This is particularly interesting in the case of isovalerylcarnitine (C5), the elevation of which can indicate the metabolic disease isovaleric acidemia (IVA). In IVA, isovaleric acid accumulates in the metabolism during the breakdown of leucine. This is the case when the enzyme isovaleryl-CoA dehydrogenase is either missing or not active enough. Isovaleric acid is removed from the metabolic cycle and excreted as isovalerylcarnitine or isovalerylglycine. The disease is noticeable in the NGS because the concentration of C5 is greatly increased. However, C5 can also be elevated if the newborn's mother treats a urinary tract infection with the antibiotic pivmecillinam before birth, resulting in pivaloylcarnitine before excretion. Pivaloylcarnitine is also detected as C5 in the NGS. C5 elevations are clarified using the IVA-second tier test. In our laboratory, 69 patients with a $\mathrm{C} 5$ elevation have been analysed since the introduction of the IVA-2nd-tier test in November 2020 until today (2021-08-18). Among them were 60 patients with the detection of pivaloylcarnitine, degradation product of the antibiotic pivmecillinam and 5 patients diagnosed with IVA. Of the 5 IVA patients, the disease was confirmed in 3 patients during a check-up and the other two were premature babies whose metabolism normalised.

\section{CONCLUSIONS}

In the case of a real IVA, the sick child must be treated as soon as possible. In the case of pivmecillinam, parents should not be unnecessarily alarmed by a false positive result for IVA, because the antibiotic is excreted and the C5 concentration normalises on its own. It has been shown that the 2 nd tier test helps to detect false indications of IVA and does not place an unnecessary burden on parents. 
Neonatal and paediatric laboratory medicine, including prenatal testing

W082

INFLUENCE OF MATERNAL AGE ON THE BEHAVIOR OF BIOCHEMICAL AND ECHOGRAPHICS MARKERS IN COMBINED FIRST TRIMESTER SCREENING FOR TRISOMY 21

\author{
E. Lepe-Balsalobre $^{1}$, J. Montenegro-Martinez ${ }^{1}$, D. NuÑez-Jurado ${ }^{1}$, J.M. Guerrero-Montavez ${ }^{1}$ \\ ${ }^{1}$ Virgen del Rocío Hospital, Sevilla.
}

\title{
BACKGROUND-AIM
}

The Combined First Trimester Screening (CFTS) became generalized in the late 1990s as a strategy to estimate the probabilities that the fetus is affected by trisomy 21 (T21) or other aneuploidies. Maternal age, one of the most influential variables in the calculation of this estimate, is experiencing a growing trend due to socioeconomic conditions and development of assisted reproductive techniques.

The aim of this study was to know the behavior of biochemical and echographics markers used in the CFTS, as well as the final result of the program in women aged 40 years or older compared to pregnant women under 40 years of age.

\section{METHODS}

Retrospective and descriptive study of CFTS performed in pregnant women with single pregnancies between 2015 and 2020. Free beta fraction of human chorionic gonadotropin ( $\beta-\mathrm{HCG}$ ) and plasma protein associated with pregnancy (PAPP-A) were determined in an analyzer Cobas 8000 (Roche Diagnostics). Nuchal translucency (NT) was measured by transvaginal ultrasound, following the specifications of the Fetal Medicine Foundation. For the risk calculation, SiPACAC software was used. Corrected median multiples (MoM) of $\beta-H C G$, PAPP-A, and TN were calculated. The final results were assessed based on the detection rate (DR) and the false positive rate (FPR).

\section{RESULTS}

The $<40$ years old group had a mean age of 29.15 years and a weight of $63.15 \mathrm{Kg}$. Results were: NT MoM=0.8963, $\beta \mathrm{HCG}$ $M o M=1.3265$ and PAPP-A MoM=1.1985. $D R=74.6 \%$ and $F P R=3.4 \%$.

The $\geq 40$ years old group had a mean age of 43.15 years and a weight of $65.78 \mathrm{~kg}$. Results were: NT MoM=0.9658, $\beta \mathrm{HCG}$ $\mathrm{MoM}=1.3966$ and PAPP-A MoM=1.2419. $\mathrm{DR}=100 \%$ and $\mathrm{FPR}=27.8 \%$.

\section{CONCLUSIONS}

The prevalence of T21 increases with age. Both DR and FPR are higher in the group over or equal to 40 years than in those under 40 years of age.

The MoM combination of the markers does not explain the higher DR and FPR in people over 40 years of age: MoM TN and $\beta-H C G$ are higher and would imply a greater risk, and MoM PAPP-A which would cause a lower risk.

In patients older than 40 years with positive CFTS, it would be appropriate to implement a contingent strategy with the use of non-invasive prenatal diagnostic techniques in order to reduce FPR and minimize the use of unnecessary invasive techniques. 
Poster Abstracts - EuroMedLab Munich 2021 - Munich, April 10-14, 2022 • DOI 10.1515/cclm-2021-5029

Neonatal and paediatric laboratory medicine, including prenatal testing

W083

SICKLE CELL DISEASE IN TUNISIA: A LOW COST METHOD USED IN NEONATAL SCREENING STRATEGY OVER 15 YEARS

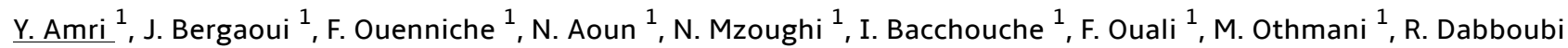

1, T. Messaoud $^{1}$

${ }^{1}$ Laboratory of Clinical Biochemistry -Children Hospital, Tunis- Tunisia

\section{BACKGROUND-AIM}

Sickle cell disease (SCD) is a severe autosomal recessive blood disorder associated with significant morbidity and mortality in early childhood. It is one of the most frequent hemoglobinopathies in Tunisia. Since simple interventions are available to prevent early fatal courses, this disease is a target condition of national neonatal screening programs in Tunisia. Here, we demonstrate the potential utility of the SCD newborn screening strategy based on isoelectric focusing (IEF).

\section{METHODS}

Samples from 71050 newborns were collected over 15 years (from 2005 to 2019) in two Tunis maternity hospitals using dried blood spot samples. A lab-prepared agarose gel for IEF was used to test these samples. The method involves casting a layer of agarose gel containing a mixture of carrier ampholytes. The blood samples are applied to the surface of the gel. The Isoelectric focusing is performed at $12^{\circ} \mathrm{C}$ at a constant power. Hemoglobin (Hb) fractions are then fixed in $15 \%$ trichloroacetic acid; the gel is washed in water, dried, and stained with a specific Hb colorant. Parents of subjects, who were found to have $\mathrm{Hb}$ abnormalities, were invited for a confirmation hemoglobin electrophoresis test using HighPerformance Liquid Chromatography (HPLC).

\section{RESULTS}

Our newborn screening programme concerns 4750 babies per annum. Overall, $1.2 \%$ of the newborns screened positive for SCD. Hemoglobin electrophoresis profiles found were as follows: 70013 (98.5\%) AA (normal), 905 (1.27\%) AS, 72 (0.1\%) AC, 29 (0.04\%) AO, 16 (0.02\%) AG, 5 (0.007\%) SS, 3 (0.004\%) AD, SO, CC and 1 (0.001\%) SG. About 210 (23\%) couples were found at risk of transmitting SCD. The IEF test detected the presence of HbS with $91.5 \%$ sensitivity and $85.7 \%$ specificity, relative to HPLC. To the best of our knowledge, the only unavoidable case of a false positive results from the $\mathrm{HbS} /$ hereditary fetal $\mathrm{Hb}(\mathrm{HPFH})$. The use of the IEF in newborn screening process could have excluded approximately $98 \%$ of all newborns from expensive confirmatory testing by HPLC.

\section{CONCLUSIONS}

The Tunisian neonatal screening strategy uses IEF as the primary technique and HPLC as the secondary technique for confirmation and for suspect profiles, and when necessary, genetic analyses as tertiary techniques for mutation screening. 
Neonatal and paediatric laboratory medicine, including prenatal testing

W084

EVALUATION OF ANALYTICAL PERFORMANCE OF AN AUTOMATIZED SOLUTION FOR THE MEASUREMENT OF ALPHAGLUCOSIDASE CONCENTRATION IN HUMAN SEMINAL PLASMA

\author{
M. Sanchez $^{1}$, S. Fernandez $^{1}$, I. Lopez ${ }^{1}$, M.A. Larraga ${ }^{2}$, M. Fite ${ }^{1}$, R. Perez ${ }^{1}$ \\ ${ }^{1}$ BioSystems S.A., Barcelona, Spain \\ ${ }^{2}$ Reference Laboratory, Hospitalet de Llobregat, Spain
}

\title{
BACKGROUND-AIM
}

Neutral alpha-Glucosidase is an enzyme considered a biochemical indicator of epididymal function. Most of the alphaglucosidase in semen corresponds to the neutral isoenzyme. The absence or decrease in its catalytic concentration may be a sign of a complete or partial obstruction located between the epididymis and the ejaculatory ducts.

BioSystems has developed an automatized solution for the measurement of Alpha-Glucosidase in seminal plasma. The assay is carried out at a neutral pH for a very short period of time, which is where the neutral isoenzyme presents its optimal activity unlike the acid form. The assay is based on enzymatic reactions that generate a coloured product that can be measured at $505 \mathrm{~nm}$. The aim of this study is the evaluation of analytical performance of this new assay.

\section{METHODS}

The assay has been applied to automated BioSystems analyzers in combination with exclusive reference materials. Detectability, linearity and precision were evaluated according to the Clinical and Laboratory Standards Institute guidelines. Correlation by linear regression analysis, and the sensitivity and specificity were determined using a commercially available method for neutral alpha-Glucosidase traceable to the WHO method. The Homogeneity of the reference materials was studied measuring the concentration of the different vials, and the stability was evaluated after storing at $4{ }^{\circ} \mathrm{C}$ after reconstitution.

\section{RESULTS}

The obtained measuring range was $2.5-80 \mathrm{mIU} / \mathrm{mL}$. Total precision (CV) was $<4.0 \%$. High correlation was obtained in the comparative study with 82 samples $(y=0.98 x-1.36 ; r=0.982)$ and the sensitivity and specificity were $100 \%$ and $91 \%$, respectively $(n=185)$. The homogeneity for the Standard and the Control were $<3.5 \%$ and both materials were stable after 7 days after reconstitution.

\section{CONCLUSIONS}

The BioSystems alpha-Glucosidase solution shows optimal detectability, linearity, precision and good correlation in the comparison with a commercially neutral alpha-Glucosidase assay. The assay has been successfully applied to automated BioSystems analyzers of different throughput and the reference materials are homogenous and stables. Overall, the analytical performance of the assay demonstrates its suitability for clinical use. 
Poster Abstracts - EuroMedLab Munich 2021 - Munich, April 10-14, 2022 • DOI 10.1515/cclm-2021-5029

Neonatal and paediatric laboratory medicine, including prenatal testing

W085

CAN SERUM OSMOLALITY BE CALCULATED IN SMALL CHILDREN?

J. Berska $^{1}$, J. Bugajska ${ }^{1}$, K. Sztefko $^{1}$

${ }^{1}$ Department of Clinical Biochemistry, Institute of Pediatrics, Jagiellonian University Medical College, Krakow, Poland

\section{BACKGROUND-AIM}

Serum osmolality is important to assess electrolyte balance affecting homeostasis of the body. Serum osmolality can be measured $\left(\mathrm{O}_{\text {meas }}\right)$ or calculated $\left(\mathrm{O}_{\text {cal }}\right)$. Many formulas for $\mathrm{O}_{\text {cal }}$ have been already published but data regarding the best equation for osmolality calculation in children and clear recommendation for using any given formula in small babies is not available. The aim of the study was to compare $\mathrm{O}_{\text {meas }}$ and $\mathrm{O}_{\text {cal }}$ using different formulas in newborns and small children.

\section{METHODS}

The study included 280 routine serum samples from children from under one month to two years in which concentration of sodium ( $\mathrm{Na}$ ), potassium (K), glucose (Glu) and urea (Urea) and osmolality (800 CLG osmometer) were measured. Osmolality was also calculated by formulas: $\mathrm{O}_{\text {cal1 }}=2 * \mathrm{Na}+\mathrm{Glu}+\mathrm{Urea}, \mathrm{O}_{\text {cal } 2}=2 * \mathrm{Na}+\mathrm{Glu}, \mathrm{O}_{\text {cal } 3}=2 *(\mathrm{Na}+\mathrm{K})+\mathrm{Glu}$ + Urea, $\mathrm{O}_{\mathrm{cal} 4}=1.86 *(\mathrm{Na}+\mathrm{K})+\mathrm{Glu}+$ Urea $+10, \mathrm{O}_{\mathrm{cal} 5}=1.89 * \mathrm{Na}+1.38 * \mathrm{~K}+1.08 * \mathrm{Glu}+1.03 *$ Urea+7.45, $\mathrm{O}_{\text {cal6 }}=1.86 *(\mathrm{Na}+\mathrm{K})+1.15 * \mathrm{Glu}$ + Urea $+14, \mathrm{O}_{\text {cal } 7}=1.09 *(1.86 * \mathrm{Na}+\mathrm{Glu}+$ Urea $), \mathrm{O}_{\text {cal } 8}=1.86 * \mathrm{Na}+\mathrm{Glu}+$ Urea +9 . Comparison of serum $\mathrm{O}_{\text {meas }}$ and $\mathrm{O}_{\text {cal }}$ was performed taking into account all samples and separately samples taken from babies $<1$ months ( $N=47)$, between $1-3$ months $(\mathrm{N}=48)$, from 3 months to 1 year $(\mathrm{N}=96)$ and between 1 and 2 years of life $(\mathrm{N}=89)$.

\section{RESULTS}

The mean value of $\mathrm{O}_{\text {meas }}\left(285.8 \pm 5.1 \mathrm{mOsm} / \mathrm{kgH}_{2} \mathrm{O}\right)$ was significantly different as compare to the mean values of $\mathrm{O}_{\text {cal }}$ $(\mathrm{p}<0.01)$ for all formulas except Ocal obtained by formula no 6 . Bland-Altman analysis showed bias between $\mathrm{O}_{\text {meas }}$ and $\mathrm{O}_{\text {cal }}$ for all tested formulas (negative bias) except for formula no 3 (positive bias). Linear regression analysis showed high correlation between $\mathrm{O}_{\text {meas }}$ and $\mathrm{O}_{\text {cal }}$ ( $\mathrm{r}$ range $\left.0.6350-0.7173, \mathrm{p}<0.0001\right)$ and similar $95 \%$ confidence interval $(\mathrm{Cl})$, regardless the formula used for calculation of $\mathrm{O}_{\text {cal }}$. The mean value of difference between $\mathrm{O}_{\text {meas }}$ and $\mathrm{O}_{\text {cal }}$ obtained by formula number 8 had the highest value in comparison with other formulas used $\left(-15.2 \mathrm{mOsm} / \mathrm{kgH}_{2} \mathrm{O}\right)$. When method comparison was performed according to age groups, Bland-Altman analysis as well as linear regression analysis did not show any other result as the ones obtained when samples from all children were used for calculation.

\section{CONCLUSIONS}

The most popular formula used for osmolality calculation should be used with care in children under two years of age due to possibility of high negative bias. 
Poster Abstracts - EuroMedLab Munich 2021 - Munich, April 10-14, 2022 • DOI 10.1515/cclm-2021-5029

Neonatal and paediatric laboratory medicine, including prenatal testing

W086

VITAMINE D AND PHOSPHOCALCIC STATUS IN MAJOR HEMOGLOBINOPATHY

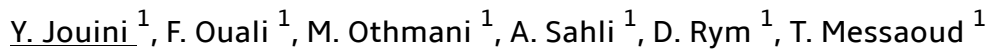

${ }^{1}$ Laboratory of Clinical Biochemistry -Children Hospital, Tunis- Tunisia

\section{BACKGROUND-AIM}

Vitamin D deficiency is growing to a large extent today, it is becoming a global health concern. In a population affected by major hemoglobinopathy: sickle cell disease (SCD), and major/intermediate thalassemia, vitamin D deficiency seems to be highly prevalent.This study aims to establish the phospho-calcic and vitamin D status in major hemoglobinopathic patients, and to evaluate its relationship with anthropometric and clinical characteristics.

\section{METHODS}

The study was processed from January 2019 until June 2019 in Laboratory of Clinical Biochemistry -Children Hospital, Tunis.

A total of 64 patients, including 28 males and 36 females, with a mean age of $17.7 \pm 6.8$ years were enrolled. A control population was selected, including 63 patients apparently healthy and with no hemoglobinopathy.

Vitamin D (Roche Elecsys Roche Diagnostics), calcium, phosphorus and other biochemical parameters (ALP, PTH, Ferritin...) were measured. Anthropometric, epidemiological and clinical information were collected. All patients have had a complete blood count as well as hemoglobin electrophoresis (Variant II BioRad).

\section{RESULTS}

The prevalence of vitamin D deficiency and insufficiency in patients with major hemoglobin disease is $54.8 \%$ and $23.4 \%$ respectively which are significantly higher than the control population $(p<0.001)$.

Vitamin D deficiency was associated with age,sex, socio-economical levels $(p<0.001)$. However, no phototype, or living zone (rural/urban) related differences were observed ( $p=0.07, p=0.195$ respectively). Hemoglobin and ferritin levels, hemoglobin phenotype, and vaso-occlusive seizures had also been correlated to vitamin D deficiency in our selected population.

Vitamin D deficiency is highly prevalent in patients with hemoglobinopathy. This may be related to inadequate nutritional status, post-transfusion hemochromatosis and vitamin $D$ receptor polymorphism.

In patients with SCD, the deficiency is correlated to vaso-occlusive seizures and hemolysis.

\section{CONCLUSIONS}

Patients with major hemoglobin disease have an increased risk of developing vitamin D deficiency. Therefore, it is necessary to monitor and detect the deficiency early in order to improve the quality of life. 
Poster Abstracts - EuroMedLab Munich 2021 - Munich, April 10-14, 2022 • DOI 10.1515/cclm-2021-5029

Neonatal and paediatric laboratory medicine, including prenatal testing

W087

INFANTILE HYPOPHOSPHATASIA IN TUNISIAN CHILD: A CASE REPORT.

$\underline{\text { R. Dabboubi }}^{2}$, Y. Amri $^{2}$, Y. Jouini $^{2}$, M. Khemiri $^{1}$, T. Messaoud $^{2}$

${ }^{1}$ Department of Pediatrics, Bechir Hamza Children's Hospital, Bab Saadoun Square, 1007, Tunisia

${ }^{2}$ Laboratoire de Biochimie Clinique et de Biologie Moléculaire «LRO0SP03», Hôpital d'Enfants Béchir Hamza, Tunis, Tunisia

\section{BACKGROUND-AIM}

Hypophosphatasia is a rare inherited disorder characterized by defective bone and teeth mineralization. Clinical manifestations of hypophosphatasia are extremely variable. Infantile hypophosphatasia is a severe form of the disease inherited as an autosomal recessive.

We report a case of infantile hypophosphatasia (HPP), which was misdiagnosed as nutritional rickets. By Sanger sequencing of the ALPL gene, the patient was homozygous for the c.896 T>C mutation.

\section{METHODS}

A 4-month-old Tunisian female baby born from first-degree consanguineous marriage originating from the south of Tunisia. She did not show any prenatal problem. Rickets prevention was done from birth correctly. She was admitted to our hospital with the chief complaint of short stature. Upon admission, physical examination for the patient showed rachitic changes. Radiological skeletal survey showed osteopenia.

At the age of 6 months, the diagnosis of HPP was confirmed biochemically and with TNSALP mutation screening. All exons including exon-intron boundaries of the TNSALP gene were amplified by PCR and sequenced.

\section{RESULTS}

Laboratory tests revealed high total calcium, high phosphorous, low alkaline phosphatase, normal parathyroid hormone, and low 25-hydroxyvitamin D.

On other hand, the patient's clinical presentation, age of diagnosis, the very low level of serum ALP activity, hypercalcemia and hyperphosphatemia suggested the diagnosis of infantile HPP associed with the vitamin D deficiency rickets.

At the age of 18 months, she was admitted for pneumonia. The clinical examination revealed an enlargement of the anterior fontanelle, rachitic deformities despite vitamin D3 supplementation. Radiographic findings show hypomineralisation. A premature loss of deciduous teeth was noted.

The patient was diagnosed as having a HPP. DNA sequencing revealed the presence of a previously reported homozygous mutationT to C transition at nucleotide position c.896 in exon 9 of TNSALP gene leading to 282 Leu > Pro substitution. This smutation was detected in the heterozygous state in the parents.

\section{CONCLUSIONS}

This case highlights the importance of association clinical, laboratory, and radiographic findings in the diagnostic of rare entities such as hypophosphatasia. Mutation analysis of ALPL gene is always necessary to confirm the diagnosis of HPP. 
Neonatal and paediatric laboratory medicine, including prenatal testing

W088

PERFORMANCE EVALUATION OF THE ADVIA CENTAUR PLGF AND SFLT-1 ASSAYS*

J. Broomfield $^{1}$, J. Mason ${ }^{2}$, T. James $^{2}$, S. Oddy $^{1}$

${ }^{1}$ Cambridge University Hospitals NHS Trust

${ }^{2}$ Oxford University Hospitals NHS Trust

\section{BACKGROUND-AIM}

Preeclampsia (PE), a disease found in pregnant women, is characterised by hypertension and proteinuria. Rapid, accurate diagnosis of PE is essential for best clinical practice and maternal/fetal care. PIGF and sFlt- 1 , both produced by the placenta, regulate fetal angiogenesis. The sFIt-1/PIGF ratio becomes elevated in PE; therefore, this ratio is valuable in helping diagnose PE. Siemens Healthineers (Tarrytown, NY, U.S.) has developed ADVIA Centaur ${ }^{\circledR}$ PE assays* for detecting PIGF and sFIt-1 in serum and plasma to be used in tandem for a clinically relevant ratio of diagnostic value. This study evaluated the precision of the new ADVIA Centaur PIGF and sFIt-1 assays. Method comparisons were also conducted between the ADVIA Centaur sFIt-1 and PIGF assays and the Roche ELECSYS sFIt-1 and PIGF assays.

\section{METHODS}

The ADVIA Centaur PIGF and sFIt-1 are sandwich assays using monoclonal anti-PIGF or anti-sFIt- 1 antibodies. A sample is added to the reaction cuvette followed by a lite reagent (monoclonal anti-PIGF or anti-sFIt-1 antibodies labeled with acridinium ester) and solid phase (monoclonal anti-PIGF or anti-sFlt-1 antibodies linked to a paramagnetic particle). Following incubation, a wash step, and magnetic separation, acidic and basic reagents are added to the reaction mixture and the resulting chemiluminescence is measured. Time to first result is 28 minutes for PIGF and 18 minutes for sFIt-1. Assay precision was assessed using five replicates each of low and high concentration controls run two times/day for five days $(n=50)$ on the ADVIA Centaur system. For the method comparison analyses 150 clinically relevant samples were used to compare the ADVIA Centaur and Roche sFIt-1 and PIGF assays. Clinical agreement with Roche using the sFIt-1/PIGF ratio was also evaluated.

\section{RESULTS}

The two levels of controls tested had mean levels found to be within their respective expected ranges. Within-lab imprecision (CV) for PIGF was $5.25 \%$ at $78 \mathrm{pg} / \mathrm{mL}, 4.73 \%$ at $1626 \mathrm{pg} / \mathrm{mL}$, and for sFlt- 1 was $4.19 \%$ at $822 \mathrm{pg} / \mathrm{mL}$ and $6.84 \%$ at $7660 \mathrm{pg} / \mathrm{mL}$.

Good agreement was observed between the ADVIA Centaur and Roche results using only values that fell within the measuring range of each assay; sFIt-1 15-85,000 pg/mL and PIGF 9-10,000 pg/mL $(n=150)$. Deming regression gave the following equations: PIGF $y=1.03 x-3 p g / m L ~ r=0.983$ and $s F I t-1 y=1.12 x-176 \mathrm{pg} / \mathrm{mL} r=0.882$. Using a ratio threshold of 38 to define low risk of PE demonstrated $94.7 \%$ agreement between the two systems.

\section{CONCLUSIONS}

These studies indicate that the ADVIA Centaur assays are reproducible and demonstrate good correlation with the Roche PIGF and sFlt-1 assays.

*Under development. The performance characteristics of this device have not been established. Not available for sale. Product availability will vary from country to country and will be subject to varying regulatory requirements. 
Neonatal and paediatric laboratory medicine, including prenatal testing

W089

\section{REFINING THE OXYHEMOGLOBIN DISSOCIATION CURVE FOR CLINICAL OXYGEN MANAGEMENT IN PREMATURE INFANTS}

N. Gangaram-Panday ${ }^{3}$, A. Tintu ${ }^{2}$, S. Willemsen ${ }^{1}$, D. Rizopoulos ${ }^{1}$, Y. De Rijke ${ }^{2}$, W. Van Weteringen ${ }^{3}$, I. Reiss ${ }^{3}$

${ }^{1}$ Department of Biostatistics, Erasmus MC, University Medical Center Rotterdam, Rotterdam, The Netherlands

${ }^{2}$ Department of Clinical Chemistry, Erasmus MC, University Medical Center Rotterdam, Rotterdam, The Netherlands

${ }^{3}$ Department of Pediatrics, Division of Neonatology, Erasmus MC Sophia Children's Hospital, University Medical Center Rotterdam, Rotterdam, The Netherlands

\section{BACKGROUND-AIM}

In neonatal care the main method for oxygen management is continuous measurement of the oxygen saturation using pulse oximetry $\left(\mathrm{SpO}_{2}\right)$. The gold standard and the best indicator of tissue oxygenation is the arterial partial pressure of oxygen $\left(\mathrm{PaO}_{2}\right)$. The nonlinear relation between the oxygen saturation $\left(\mathrm{sO}_{2}\right)$ and oxygen pressure $\left(\mathrm{pO}_{2}\right)$ is described by the oxyhaemoglobin dissociation curve (ODC). Oxygen management with $\mathrm{SpO}_{2}$ remains challenging due to factors influencing the ODC. This study aimed to create a model-based description of the ODC including affecting covariates, based on a large clinical data set. Additionally, the aim was to create model-based estimation of $\mathrm{PaO}_{2}$ from $\mathrm{SpO}_{2}$ levels in premature infants.

\section{METHODS}

Children aged 0 to 18 years, admitted to Erasmus MC Sophia Children's Hospital between June 2007 and June 2017, were included in this retrospective study. The nonlinear relation between $\mathrm{sO}_{2}$ and $\mathrm{pO}_{2}$ and vice versa was determined using generalised additive models (GAM). Co-variables such as $\mathrm{pH}, \mathrm{pCO}_{2}$, hemoglobin, and postnatal age and random intercepts were included in both models. Data was randomly divided into train or test data sets. Models were initially fit on the training data set and the test set was used to evaluate the fitted models. A blood gas data set including $\mathrm{SpO}_{2}$ values in infants admitted to the neonatal intensive care unit between June 2017 and June 2019 was used to validate the $\mathrm{PaO}_{2}$ estimation model on matched $\mathrm{SpO}_{2}$ levels.

\section{RESULTS}

A total of 7044 patients with 126256 blood gas samples were included for analyses. The GAM model with sO $\mathrm{S}_{2}$ as response variable had an $r$-squared $\left(R^{2}\right)$ value of 0.93 with a root mean squared error (RMSE) of 0.027 and a mean squared error (MSE) of 0.0007 in the train data set. The $\mathrm{pO}_{2}$ model had an $\mathrm{R}^{2}$ value of 0.86 , with RMSE of 0.17 and a MSE 0.03. In the validation data set the estimated $\mathrm{PaO}_{2}$ levels from $\mathrm{SpO}_{2}$ values showed an agreement (bias ( $95 \%$ limits of agreement)) of 0.2 (-2.4 to 2.8) kPa with arterial $\mathrm{PaO}_{2}$ levels and a Pearson's correlation coefficient of $r=0.85(n=468)$.

\section{CONCLUSIONS}

A new model-based method is presented for oxygen management in premature infants, which accurately estimates $\mathrm{PaO}_{2}$ values from clinical $\mathrm{SpO}_{2}$ measurements and factors influencing the ODC. 
Neonatal and paediatric laboratory medicine, including prenatal testing

W090

DEVELOPMENT AND PRELIMINARY EVALUATION OF A METHOD FOR THE DETERMINATION OF GROWTH HORMONE IN NEWBORNS ON DRIED BLOOD SPOTS

C. Vantaggiato $^{1}$, C. Giavoli ${ }^{2}$, M. Arosio ${ }^{2}$, F. Giacchetti ${ }^{2}$, A. Sharif Rouhani ${ }^{1}$, P. Di Deo ${ }^{1}$, C. Varani ${ }^{1}$, M. Modica Amore ${ }^{1}$, A. Di Modugno ${ }^{1}$, F. Napolitano ${ }^{1}$, M. Vidali ${ }^{1}$, F. Ceriotti ${ }^{1}$

${ }^{1}$ Clinical Chemistry Unit, Fondazione IRCCS Ca' Granda Ospedale Maggiore Policlinico, Milan, Italy

${ }^{2}$ Endocrinology Unit, Fondazione IRCCS Ca' Granda Ospedale Maggiore Policlinico, Milan, Italy

\section{BACKGROUND-AIM}

Severe deficiency of GH of the newborn is a rare but potentially life-threatening disease. GH can be measured during the first week of life when levels are physiologically higher (neonatal hypersomatotropism). GH evaluation using dried blood spots (DBS) may offer several advantages: easier transportation and storage, reduced costs, allowing centralization and method standardization. Aim of the study was to validate a method for measuring GH in newborns from DBS.

\section{METHODS}

Whole blood in EDTA and serum was collected from patients attending the Endocrinology Unit. $50 \mu \mathrm{L}$ of EDTA-blood was spotted onto Guthrie cards (LTA SrI) (diameter disks $13 \mathrm{~mm}$ ) which were air dried at RT for 4 hours and then processed or stored at $-20^{\circ} \mathrm{C}$ up to 2 months. 3 disks $(5.5 \mathrm{~mm})$ were punched out into a $2 \mathrm{~mL}$ polypropylene tube and $250 \mu \mathrm{L}$ of PBS $1 X$, with or without Tween-20 $(0.05 \%, 0.5 \%$ or $1 \%)$, were added. Samples were incubated at RT on an orbital shaker for 2 or 16 hours and then centrifuged at $12500 \mathrm{rpm}$ for $1 \mathrm{~min}$. GH in supernatants or undiluted sera was determined by Immulite 2000 (Siemens Healthineers).

\section{RESULTS}

Interference by hemolysis present after extraction was evaluated by spiking extracted samples with known concentrations of $\mathrm{GH}$ ( 2 experiments at 3.2 and $9.0 \mu \mathrm{g} / \mathrm{L}$ ). No interference was detected (recovery>99\%). A calibration curve was built by plotting GH measured in serum vs extracted GH ( 3 independent replicates, 8 levels from $1 \mu \mathrm{g} /$ $\mathrm{L}$ to $50 \mu \mathrm{g} / \mathrm{L})$. Recovery at each level was $>90 \%$. Linearity was verified $\left(R^{2}>0.99\right)$ up to a $\mathrm{GH}$ serum concentration of $50 \mu \mathrm{g} / \mathrm{L}$. Mean serum/DBS GH ratio was 14.4 . Repeatability at the 8 tested concentrations was $11.1 \%, 2.4 \%, 3.5 \%$, $3.5 \%, 6.1 \%, 2.9 \%, 5.2 \%, 2.7 \%$. Further precision experiments ( 6 independent replicates at $7.7 \mu \mathrm{g} / \mathrm{L}$ ) confirmed previous observations $(\mathrm{CV} \%=3.7 \%)$. No appreciable differences were found between samples stored at $-20^{\circ} \mathrm{C}$ up to 2 months or directly processed (similar serum/DBS ratio and recovery $>90 \%$ ), or between samples extracted with Tween or only PBS (at $50 \mu \mathrm{g} / \mathrm{L}$ differences $<5 \%$ ), or between samples incubated for 2 or up to 16 hours (at 2.9, 7.7 and $50 \mu \mathrm{g} / \mathrm{L} \%$ differences were $4.2 \%, 5.9 \%$ and $12.6 \%$ ).

\section{CONCLUSIONS}

Preliminary evaluation suggests that this method can be used to measure GH in newborns using DBS. 
Neonatal and paediatric laboratory medicine, including prenatal testing

W091

\section{NT-PROBNP AS A BIOMARKER OF MATERNAL COMPLICATIONS IN FIRST TRIMESTER OF PREGNANCY}

M.N. Nan ${ }^{1}$, C. Trilla-Solá ${ }^{2}$, A. Antonijuan-Parés ${ }^{1}$, V. Orantes-Gallego ${ }^{1}$, M. Grau-Agramunt ${ }^{1}$, J. Ordoñez-Llanos ${ }^{1}$, E. Llurba-Olivé $^{2}$, Á. García-Osuna ${ }^{1}$, J. Mora-Brugués ${ }^{1}$

${ }^{1}$ Department of Biochemistry, Hospital de la Santa Creu i Sant Pau, Barcelona

${ }^{2}$ Department of Obstetrics and Gynaecology, Hospital de la Santa Creu i Sant Pau, Barcelona

\section{BACKGROUND-AIM}

Preeclampsia (PE) is associated with a 2- to 4-fold increase risk of developing cardiovascular disease (CVD) in the short and long term. When PE is accompanied by small for gestational age (SGA) and/or preterm birth (PTB), the adjusted risk for CVD is increased by $45 \%$ for the next 5 years post-partum.

Also, PE and CVD share common risk factors like hypertension, renal disease and Diabetes Mellitus. CVD biomarkers have been proposed as useful tools in this context.

Natriuretic peptides, particularly type B (BNP, NT-proBNP) are sensitive to cardiac alterations existing in PE and are also predictors of its severity.

The aim of the study is to determine if NT-proBNP could predict different pregnancies complications (PE, SGA, PTB) when measured in first trimester.

\section{METHODS}

This was a retrospective case-control study of first trimester pregnancies. The case group consisted of 210 pregnant women who developed PE, SGA and / or PTB. The control group consisted of 208 pregnant women without these complications.

NT-proBNP was measured in maternal blood samples during first trimester of pregnancy using an electrochemiluminescence immunoassay.

\section{RESULTS}

NT-proBNP concentrations did not correlate with gestational weeks ( $r$ Spearman $=0.043, p=0.383$ ) or maternal age $(r$ Spearman=-0.092, $\mathrm{p}=0.060$ ).

Maternal age and gestational weeks showed no statistical significant differences between women who developed $\mathrm{PE}$, SGA or PTB and controls ( $p=0.407$ and $p=0.897$, respectively).

Significant decreased serum concentrations of NT-proBNP $(\mathrm{p}<0.001)$ were seen in PE, SGA and PTB women (median $36.80 \mathrm{ng} / \mathrm{L}, 55.78 \mathrm{ng} / \mathrm{L}$ and $44.79 \mathrm{ng} / \mathrm{L}$, respectively) compared to controls (median $68.71 \mathrm{ng} / \mathrm{L}$ ).

ROC curves were performed to determine whether NT-proBNP could discriminate pregnancy complications. NT-proBNP cut-off value for the prediction of PE was $27.4 \mathrm{ng} / \mathrm{L}$ (area under the curve [AUC] 0.730; sensitivity $31.4 \%$; specificity $89.9 \%$ ), $27.4 \mathrm{ng} / \mathrm{L}$ for SGA (AUC 0.600; sensitivity $19.6 \%$; specificity $90.7 \%$ ) and $26.7 \mathrm{ng} / \mathrm{L}$ for PTB (AUC 0.670; sensitivity $22.0 \%$; specificity $89.4 \%)$.

\section{CONCLUSIONS}

NT-proBNP concentrations are a moderate predictor of PE, SGA, and PTB. The lower concentrations of NT-proBNP in the first trimester of pregnancy could promote the development of placental and/or hypertensive complications in pregnant women. 
Neonatal and paediatric laboratory medicine, including prenatal testing

W092

EVALUATION OF PRE-ANALYTICAL STABILITY AND DETERMINATION OF A CUTOFF VALUE FOR UMBILICAL CORD TOTAL BILIRUBIN TO PREDICT SIGNIFICANT NEONATAL HYPERBILIRUBINEMIA

\author{
L. Vandervore $^{1}$, K. Eeckhout $^{1}$, S. Weekx ${ }^{1}$, K. Ver Elst $^{1}$, A. Meskal ${ }^{1}$, K. Plaskie $^{2}$, S. Vermeiren ${ }^{1}$ \\ ${ }^{1}$ Department of Laboratory Medicine, GZA hospitals, Oosterveldlaan 24, 2610 Antwerp, Belgium \\ ${ }^{2}$ Department of Neonatology, GZA hospitals, Oosterveldlaan 24, 2610 Antwerp, Belgium
}

\title{
BACKGROUND-AIM
}

Post-natal monitoring of total bilirubin is important to prevent clinical complications of neurotoxic hyperbilirubinemia. However, neonates without risk factors may be discharged before 72 hours when potential hyperbilirubinemia might still develop prior to planned follow-up. Especially during the COVID-19 pandemic, early discharge was seen more often, underlining the need for an early biomarker. Here, neonatal umbilical cord bilirubin (UCB) was studied to predict development of significant neonatal hyperbilirubinemia.

\section{METHODS}

Venous UCB was systematically gathered during 4 months at the maternity wards of GZA hospitals. For each case, two lithium heparin tubes were filled and total bilirubin was determined (Alinity c, Abbott, USA). 1003 neonates were included and patient data were retrospectively analyzed. Pre-analytical stability was evaluated between different subgroups. Predictive value of UCB was studied with ROC-analysis.

\section{RESULTS}

UCB results were significantly decreased in the total cohort when measured in light-exposed (H1) versus dark (HD) lithium heparin $(P=0.0258)$. Significant photolysis depended on average UCB concentration, with lower concentrations being more susceptible (a term, $\mathrm{P}=0.0224$; $\mathrm{ABO}$ compatible, $\mathrm{P}=0.0231$; DAT negative, $\mathrm{P}=0.0207$ ). However, light exposure only led to a mean degradation of $-0.1 \%$ and did not seem to affect predictive value significantly (AUC UCB $H 1=0.721$ versus $A U C U C B H D=0.726$; $P=0.2542$ ). $U C B$ proved to be a significantly better predictive marker of phototherapy than $A B O-i n c o m p a t i b i l i t y ~(A U C=0.597)$ or DAT positivity ( $A U C=0.568)$. UCB screening cutoff was $1.6 \mathrm{mg} /$ $\mathrm{dL}$ in dark conditions and $1.5 \mathrm{mg} / \mathrm{dL}$ when exposed to light. Post-test probability at these cutoffs were 11.4 and $11.0 \%$. Diagnostic cutoff was not altered by light-exposure $(2.6 \mathrm{mg} / \mathrm{dL})$ and post-test probabilities were $33.3 \%(\mathrm{H} 1)$ and $36.1 \%$ (HD). Prevalence of significant hyperbilirubinemia was $9.57 \%$ within our total cohort.

\section{CONCLUSIONS}

This study shows that UCB measurement could aid in the prediction of neonatal hyperbilirubinemia and should be included in standard post-natal monitoring guidelines. Although total bilirubin is known to be photo-sensitive, we showed that light exposure did not affect predictive value of UCB. 
Neonatal and paediatric laboratory medicine, including prenatal testing

W093

POCT DETERMINATION OF NEONATAL CAPILLARY BILIRUBINEMIA USING BILISTICK@: ANALYTICAL PERFORMANCES AND CLINICAL VALUE

\author{
A. Mailloux $^{1}$, F. Khettab ${ }^{1}$, S. Huguet-Jacquot ${ }^{1}$, H. Delaby $^{1}$, J. Beaud ${ }^{1}$, C. Toly-Ndour ${ }^{1}$, M. Vaubourdolle $^{2}$ \\ ${ }^{1}$ Centre National de Référence en Hémobiologie Périnatale (CNRHP), Hospital Saint-Antoine, AP-HP, Paris, France \\ ${ }^{2}$ Départment of Biochemistry, DMU BioGeM, Hospital Saint-Antoine, AP-HP, Paris, France
}

\title{
BACKGROUND-AIM
}

In pediatrics, accurate measurement of total serum bilirubin (TSB) is of major importance for reliable diagnosis and appropriate management of neonatal jaundice. This TSB assay should be available quickly for a safe management of newborn jaundice. Today equipment for point-of-care and laboratory testing perform bilirubin measurement. The follow-up of jaundice is done in real-time either using non-invasive transcutaneous bilirubin meters (BTC) or capillary blood POCT devices (involving CO-Oximetry or photometry). Several studies evidenced poor comparability of results obtained with the different methods. To progress, we assess the analytical performances of Bilistick $\AA_{\text {, a POCT device }}$ using photometric estimation of bilirubin on capillary blood, and we compared results both to central lab expected values and to thorax-measured BTC.

\section{METHODS}

50 consecutive samples, addressed to the CNRHP lab for follow-up of neonatal jaundice, were measured either with diazo reaction method on an Indiko ${ }^{\circledR}$ analyzer (plasma), or using Bilistick ${ }^{\circledR}$ (Bilimetrix, Italy) device (total blood and plasma). Two control samples (200 and $270 \mu \mathrm{mol} / \mathrm{L}$ bilirubin concentrations) were used for imprecision studies. Bilistick ${ }^{\circledR}$ performances (repetability, accuracy) were estimated using CLSI-based protocols. Practicability was assessed for use in POCT situation.

\section{RESULTS}

Analytical acceptance limits at high bilirubin levels ( $>150 \mu \mathrm{mol} / \mathrm{L}$ ) were for imprecision a maximum CV $8 \%$ and for accuracy $15 \%$ limit bias. These limits integrate the guidelines for diagnosis and monitoring of neonatal jaundice. Results show that Bilistick ${ }^{\circledR}$ gave acceptable results for imprecision studies. Compared to expected central lab results, a mean bias of $-15 \mu \mathrm{mol} / \mathrm{L}$ was found when blood sample were tested. This bias was reduced in plasma samples. A major positive bias was found in case of hemolysis but the device was able to detect this hemolysis in 5/6 cases. We compared the results between plasma and serum and between BIlistick ${ }^{\circledR}$ and BTc. These data confirmed the underestimation of BIlistick ${ }^{\circledR}$ in blood, probably linked to a light diffusion with RBC not corrected by a recalibration in silico. Practicability study (easy-to-use, small, cheap) showed that Bilistick ${ }^{\circledR}$ can be used in POCT situation for phototherapy monitoring in clinical settings where neither BTc device nor POCT CO-oximeter is available.

\section{CONCLUSIONS}

Our study allowed us to increase our knowledge about analytical performances of routinely used bilirubin tests, to progress in harmonization process for neonatal bilirubin. The added value of the clinical lab will be an adequate interpretation of results using published bilirubin nomograms for identification and monitoring of neonatal hyperbilirubinemia. 
Poster Abstracts - EuroMedLab Munich 2021 - Munich, April 10-14, 2022 • DOI 10.1515/cclm-2021-5029

Neonatal and paediatric laboratory medicine, including prenatal testing

W094

DIFFERENCES IN THE SERUM LEVELS OF TROPONINE T AND TROPONINE I IN PEDIATRIC PATIENTS WITH NEUROMUSCOLAR DISEASES

M. D'Agostini $^{1}{ }^{1}$, F. Albanese ${ }^{1}$, F. Gazzotti $^{1}$, R. Luciano ${ }^{1}$, F. Giustini ${ }^{1}$, O. Porzio ${ }^{1}$

${ }^{1}$ Clinical Laboratory, Bambino Gesù Children's Hospital, IRCCS, Rome, Italy

\section{BACKGROUND-AIM}

The troponine complex is expressed in the muscle cells and it is involved in the regulation of muscle contraction. Several subunits are known including C, I and T. Troponine C (TnC) binds Ca2+ while Troponin I (TnI) inhibits the ATPase activity of actomyosin complex. Troponine $\mathrm{T}(\mathrm{TnT})$ interacts with tropomyosine molecules.

Increased serum levels of Troponine I and T are associated with pathological conditions such as AMI (acute myocardial infarction). In non-ischemic cardiac injuries including myocarditis, heart failure and other cardiomyopaties an increase of troponine levels can also occur.

In patients with neuromuscolar diseases without cardiomyopathy an increase of serum levels of Troponine $T$ but not Troponine I was observed. Further investigation is required to identify the cause of these alterations. However, several hypotheses were proposed: 1) A cross reaction of cardiac TnT antibodies with skeletal TnT; 2) Re-expression of fetal TnT for process regeneration due to chronical tissue damage; 3) Myopathies with concomitant cardiac involvement; 4) Structural heart injury not related to skeletal muscle diseases.

\section{METHODS}

In our study, we evaluated serum TnT and TnI in 27 pediatric patients affected by different neuromuscolar diseases: SMA type 1, SMA type 2, SMA type 3, Duchenne Muscolar Dystrophy (DMD) and Mitochondrial Encephalopathy. TnT and TnI were evaluated by Elecsys Troponin T, Cobas c8000 platform Roche Diagnostics (ECLIA) and Abbot High-Sensitivity TnI, Alinity platform, Abbott Diagnostics (CLIA), respectively.The URL (upper reference limit) of hs-TnT is $13 \mathrm{ng} / \mathrm{L}$ with a CV of $10 \%$. For the hs-TnI the URL is $26,2 \mathrm{ng} / \mathrm{L}$ with a CV of $4 \%$.

\section{RESULTS}

About $85 \%$ of patients ( 23 of 27 ) displayed high level of TnT with normal or under the URL values of TnI $(82.43 \pm 15.72$ compared to $7.00 \pm 3.40$ respectively, $\mathrm{p}<0.0001$ ). Only 3 patients showed low levels of both Troponines, while a patient with DMD was characterized by increased TnI and TnT values.

\section{CONCLUSIONS}

In conclusion, we demonstrated the relevance to measure cardiac TnI in patients with neuromuscolar diseases for the accurate monitoring of the cardiac condition. 